\title{
La « culture scientifique » dans les ZEP
}

\section{Marcel Georges}

\section{OpenEdition}

\section{Journals}

Édition électronique

URL : http://journals.openedition.org/ries/3352

DOI : 10.4000/ries.3352

ISSN : 2261-4265

\section{Éditeur}

Centre international d'études pédagogiques

\section{Édition imprimée}

Date de publication : 1 juin 1997

Pagination : 81-84

ISSN : 1254-4590

\section{Référence électronique}

Marcel Georges, «La " culture scientifique » dans les ZEP », Revue internationale d'éducation de Sèvres [En ligne], 14 | 1997, mis en ligne le 30 juillet 2013, consulté le 21 avril 2019. URL : http:// journals.openedition.org/ries/3352 ; DOI : 10.4000/ries.3352

Ce document a été généré automatiquement le 21 avril 2019

(c) Tous droits réservés 


\title{
La « culture scientifique » dans les ZEP
}

\author{
Marcel Georges
}

\section{NOTE DE L'AUTEUR}

Le collège René Cassin de Chanteloup-les-Vignes est situé en zone d'éducation prioritaire, compte tenu de son environnement socioéconomique et socioculturel. Il accueille 428 élèves (et 69 élèves dans la section d'enseignement général professionnel adapté) de 25 nationalités différentes. Voir également p. 73 la note sur les ZEP.

1 Les regroupements souvent homogènes de la population scolaire engendrent contrastes et disparités. Dans certaines aires géographiques aisées ou favorisées, les jeunes sont programmés, dès leur naissance, aux plus hautes fonctions et sont sous le coup de «l'overdose » scientifique, gage de réussite pour faire partie de la «classe dirigeante ». Course infernale aux concours et aux diplômes, le tremplin étant principalement les mathématiques. Il faut croire que, dans certains milieux, la culture scientifique est une seconde nature. Mais, ailleurs, qu'en est-il de tous les autres qui ne sont guère attirés par les disciplines scientifiques et les options technologiques, malgré les encouragements de toutes sortes prodigués par le ministère de l'Éducation nationale?

Il ne s'agit pas dans ces lignes de remettre en question les programmes nationaux, mais bien de présenter des démarches pédagogiques capables de redonner aux jeunes le goût des sciences et des mathématiques. Force est de constater que des moyens supplémentaires sont nécessaires à la survie des projets qui favorisent la culture scientifique. C'est le cas des zones d'éducation prioritaires et des établissements classés «sensibles». On ne décrit plus les multiples problèmes que connaissent les écoles et collèges des ZEP, les difficultés économiques liées au chômage, les familles éclatées, les différences de mentalités, de culture et de croyance, l'absence d'éducation et le manque de motivation chez beaucoup de jeunes on ne décrit plus les problèmes de violence dans lesquels se débattent les établissements en ZEP. C'est ainsi que l'école et le collège ont 
tendance à prendre le relais des familles pour éduquer et former. Mais cela ne se fait pas sans enthousiasme, sans dévouement que les difficultés entament parfois.

Parallèlement aux moyens mis en œuvre, il y a la volonté unanime des enseignants de relever le défi par la recherche de nouveaux axes pédagogiques. Je voudrais ici, à titre d'exemple, évoquer les expériences de la ZEP et en particulier les expériences du collège René-Cassin de Chanteloup-les-Vignes. Il s'agit bien de défi et d'innovation tranquille. La préoccupation essentielle des ZEP a toujours été la maîtrise de la langue orale et écrite, parfois au détriment des projets scientifiques et techniques. Cette lacune est aujourd'hui partiellement comblée.

Dans la ZEP de Chanteloup, les relations entre les différents niveaux scolaires sont particulièrement dynamiques. Le collège a mis à la disposition des enseignants et des élèves du cycle 3 de l'élémentaire non seulement les salles de sciences mais aussi la médiathèque de l'établissement. Instituteurs et professeurs ont élaboré des séquences pédagogiques communes, testées dans les classes du primaire, comme au collège par permutation des enseignants. Il va de soi que la confrontation des expériences par le rapprochement des équipes pédagogiques favorise la recherche, l'objectif principal étant, par la mise en commun des stratégies, la remobilisation de l'intelligence des jeunes. Lourde tâche, cette remobilisation. Car elle s'oppose à l'ignorance d'un trop grand nombre de familles qui, confrontées aux difficultés quotidiennes de la vie, méconnaissent la culture scientifique et technique.

D'un autre point de vue, c'est également un atout. Les ZEP sont quelque peu un «terrain vierge » qui se prête à toutes les expériences pédagogiques. L'essentiel est la réussite quand on a à traiter des élèves totalement ou partiellement démotivés, des élèves d'une autre «planète » à qui il faut tout apprendre pour les placer - ou replacer - dans notre monde. Que d'innovations pour y arriver!

6 La remotivation par le biais de projets spécifiques, hors du champ disciplinaire ordinaire et de ses contraintes, permet d'accéder librement à cette culture scientifique. Ce début de réussite, combien modeste, on le doit dans les collèges en ZEP aux moyens horaires et humains supplémentaires mis à la disposition des établissements. Je n'ose imaginer ce que seraient les collèges sans ces moyens. En effet - et il faut le dire - le «quadrillage pédagogique » de la population scolaire est seul capable d'éviter les explosions, facettes négatives du désespoir et la "ghettoïsation" des ZEP. L'encadrement humain est primordial. Être toujours plus près, plus attentif aux problèmes des jeunes, c'est redonner confiance et surtout le goût d'aller au bout des projets par l'apprentissage de l'effort, et le plaisir de réussir. La dure réalité des ZEP a mis en évidence le travail en groupe des enseignants qui stabilise et sécurise. Les projets sont pour la plupart multidisciplinaires et concourent à l'épanouissement des élèves. La présence de deux ou trois enseignants pour encadrer une classe ou un groupe lors de séquences hebdomadaires permet aux élèves de se libérer de certaines contraintes face à la connaissance, d'accéder petit à petit au savoir et d'ignorer la peur de l'inconnu qui paralyse. Cet objectif atteint, on peut espérer que l'état d'esprit né des activités pluridisciplinaires aura des effets tout à fait bénéfiques sur l'enseignement disciplinaire proprement dit. La preuve est apportée par le collège René Cassin de Chanteloup qui a déjà une longue pratique des projets multidisciplinaires. Ce sont ces mêmes projets qui ont canalisé l'énergie des jeunes et évité le pire dans leur scolarité.

7 L'option "sciences » en $4^{\mathrm{e}}$ en est un exemple. Les élèves d'une classe sous la direction de deux professeurs élaborent une brochure scientifique largement diffusée dans 
l'établissement. C'est déjà une approche de la culture scientifique et la preuve que des élèves bien guidés ont appris non seulement à maîtriser l'outil informatique qui a permis la confection d'un « magazine d'actualité scientifique » mais à rechercher des documents, rédiger et mettre en page. Les thèmes choisis montrent bien tout l'intérêt de tels projets qui font appel à l'esprit d'initiative des jeunes. Ceux-ci, débarrassés des contraintes disciplinaires et de l'obligation de résultats évaluables, donnent enfin le meilleur d'euxmêmes. Ils ont - peu ou prou - acquis des méthodes de travail et de réflexion qu'ils transposent assez naturellement par la suite dans leurs études. N'est-ce pas le but recherché? Aboutir à une autonomie certaine et faire en sorte, par une certaine « vulgarisation » que la culture scientifique devienne accessible à tous.

8 À titre d'exemple, voici les intitulés des certains thèmes étudiés :

- de la lumière à l'image,

- à la découverte des mathématiques,

- Pasteur : chimiste et biologiste,

- la médecine,

- l'évolution de la terre,

- la bionique,

- la météorologie.

9 Nous ne parlerons pas ici des autres projets multidisciplinaires artistiques tels que "Claude Monet et le paysage impressionniste », mais bien des projets tels que « Arts et techniques " qui est un atelier de créations et de constructions de décors en liaison avec d'autres projets de l'établissement, ou « Arts, mécanismes et machinerie » qui associe des professeurs d'arts plastiques, de technologie et d'atelier métallerie, l'objectif étant la réalisation d'une sculpture réalisée par les élèves du collège et de l'atelier «métaux en feuilles » de la SEGPA (Section d'enseignement général et professionnel adapté).

On peut aussi citer la réalisation d'un lexique en mathématiques qui sera repris l'année prochaine en utilisant tous les outils informatiques disponibles : tableurs, traitement de texte, logiciels graphiques et d'édition.

11 Dans le même esprit, une " plaquette sciences » consacrée aux insectes sera réalisée par une classe de $5^{\mathrm{e}}$ et deux de ses professeurs : technologie et sciences de la vie et de la terre. Cette plaquette sera entièrement traitée en informatique. Dans ce projet interdisciplinaire comme dans d'autres, l'« esprit scientifique ", incontournable, est recherché et mis sur le devant de la scène, comme dans cet autre projet «la route du sel».

12 Cet esprit scientifique se retrouve également dans "Communication et entreprise » ou dans la réalisation de la plaquette du collège René Cassin. Dans ce dernier projet, qui est reconduit par deux professeurs de sciences et de technologie, des élèves de $4^{\mathrm{e}}$ vont apprendre à maitriser les outils informatiques : PAO (publication assistée par ordinateur), CAO (conception assistée par ordinateur), DAO (dessin assisté par ordinateur). Ils vont ainsi utiliser des logiciels performants : Works, Publisher, Excel, des logiciels de dessin. Ils apprendront aussi la prise et le traitement des photos numériques.

13 Ce foisonnement de projets sans cesse renouvelés montre bien que les moyens dont bénéficient les ZEP stimulent l'imagination, loin des sentiers battus, et permettent en toute liberté l'investissement des jeunes. C'est aussi la spécificité du collège René Cassin qui a opté pour l'informatique et les technologies nouvelles. Les résultats d'ensemble 
peuvent paraître modestes mais, pour l'établissement, chaque petite réussite est une victoire remportée sur l'ignorance et des difficultés inhérentes aux ZEP.

Pour conclure, je voudrais citer cet autre projet important à Chanteloup-Les-Vignes : le jumelage ZEP-Musée, jumelage avec le Parc aux étoiles, l'observatoire de Triel. Un projet commun entre les deux collèges de la ville (René-Cassin et Magellan), basé sur l'astronomie, associe les professeurs de physique et leurs élèves de $4^{\mathrm{e}}$. Mais, à un autre niveau, les écoles maternelles et élémentaires sont également impliquées par le projet astronomie et l'expérimentation sciences qui vise à développer la pratique des sciences par des expériences simples. La culture scientifique et technique, par le biais de ce jumelage, est devenue un des axes importants de la politique de la ZEP de Chanteloup et complète les actions en faveur de la maîtrise de la langue.

Donner le goût des sciences à nos élèves n'est pas une chose aisée. Notre priorité est de remotiver, remobiliser l'intelligence des élèves pour leur donner l'envie d'apprendre et de réussir. Les projets pluridisciplinaires dans de nombreux domaines contribuent largement à la formation scientifique des jeunes, en relation avec l'enseignement disciplinaire proprement dit. Les équipes pédagogiques n'ont pas toujours eu la formation nécessaire à la pratique du travail en groupe mais leurs tâtonnements et les expériences réalisées en commun sont un encouragement à concevoir une autre approche de l'enseignement. Aujourd'hui, l'enseignement disciplinaire se conçoit difficilement sans projets pluridisciplinaires d'accompagnement. Ces derniers symbolisent l'esprit pédagogique nécessaire à la réussite et à la motivation des jeunes pour les technologies nouvelles.

\section{RÉSUMÉS}

Comment des projets multidisciplinaires favorisent-ils la sensibilisation d'élèves de milieu particulièrement défavorisé à une culture scientifique, en relation avec l'enseignement disciplinaire? Quelques exemples bien ciblés permettent de saisir cette démarche.

\section{INDEX}

Index géographique : France

Mots-clés : éducation scientifique, études scientifiques, sciences, zone prioritaire, curriculum

\section{AUTEUR}

\section{MARCEL GEORGES}

Principal, collège de Chanteloup-les-Vignes, France. 